\title{
Fecundidad y proporción de sexos de Panulirus inflatus en la costa occidental del Golfo de California, México
}

\author{
Fecundity and sex ratio of Panulirus inflatus in the west coast of \\ the Gulf of California, México \\ José Iván Velázquez-Abunader' ${ }^{1}$ Marcial Villalejo-Fuerte ${ }^{1}$ \\ y Arturo Tripp-Quezada ${ }^{1}$ \\ ${ }^{1}$ Instituto Politécnico Nacional, Centro Interdisciplinario de Ciencias Marinas, \\ La Paz, Baja California Sur, México. A.P. 592. C.P. 23000 \\ mvillale@ipn.mx
}

\begin{abstract}
The fecundity and sex ratio of $P$. inflatus were studied in the western coast of the Gulf of California, Mexico. From February 2004 to January 2005, 567 lobsters were captured, 302 males (M) and 265 females (F). The sex ratio was $1 \mathrm{H}: 1.13 \mathrm{M}$ which did not differ significantly from 1:1. Fecundity varied between 94,837 and 830,149 eggs (384,314 $\pm 162,147$, mean $\pm S D, n=97)$. On average, the relative fecundity was $952 \pm 222$ eggs g $^{-1}$. The Index of Reproductive Potential (IRP) showed that females with cephalotorax length (CL) smaller than $80 \mathrm{~mm}$ contributed with $10.82 \%$ of the eggs' production, probably because of their low fecundity and spawn only two times during the reproductive season. On the other hand, females between 80 and $110 \mathrm{~mm}$ CL contributed with $80.23 \%$ of the spawned eggs. These females had a high fecundity, potentially spawn up to four times during the reproductive season, and presented high frequencies of occurrence in the samples. Although females larger than 110 mm CL had a high fecundity (around 800 thousand eggs), and may spawn up to four times during the reproductive season, their contribution in eggs is low, $10.55 \%$, probably because they are not abundant in the population.
\end{abstract}

Key words: Reproductive potential, lobster

\section{Introducción}

La pesquería de langostas en el Pacífico mexicano está representada principalmente por tres especies Panulirus interruptus (Randall, 1840), Panulirus gracilis Streets, 1871 y Panulirus inflatus (Bouvier, 1895) esta última, ocupa el tercer lugar de la producción en México, registra capturas anuales sobre las 150 tm (SAGARPA 2003), es considerada como especie endémica de la costa Oeste de México, se distribuye desde Isla Magdalena en la costa occidental de la Península de Baja California, incluyendo
Resumen.- Se analizó la fecundidad y proporción de sexos de $P$. inflatus en la costa occidental del Golfo de California, México. De febrero de 2004 a enero de 2005 se capturaron 567 langostas, 302 machos (M) y 265 hembras (H). La proporción de sexos fue $1 \mathrm{H}: 1,13 \mathrm{M}$ y no difirió significativamente de 1:1. La fecundidad varió entre 94.837 y 830.149 huevos (media \pm desviación estándar $384.314 \pm$ $162.147, \mathrm{n}=97)$. La fecundidad relativa media fue de $952 \pm$ 222 huevos $\mathrm{g}^{-1}$. El Índice de Potencial Reproductivo (IPR) mostró que hembras menores de $80 \mathrm{~mm}$ de longitud cefalotorácica (LC) aportaron el 8,95\% de los huevos producidos, esto probablemente se debe a que tienen fecundidades bajas y a que desovan solamente dos veces durante la estación reproductiva. Por otra parte, las hembras entre 80 y $110 \mathrm{~mm}$ de LC aportaron el 80,23\% de los huevos desovados. Estas hembras tuvieron fecundidades altas, potencialmente desovaron hasta cuatro veces durante la temporada de reproducción y presentaron altas frecuencias de ocurrencia numérica en los muestreos. A pesar de que las hembras mayores de $110 \mathrm{~mm}$ de LC tuvieron las fecundidades mas altas (alrededor de 800 mil huevos), y pueden desovar hasta cuatro veces durante la temporada reproductiva, su aporte de huevos es bajo, 10,55\%, probablemente porque son poco abundantes en la población.

Palabras clave: Potencial reproductivo, langosta

el Golfo de California hasta el Golfo de Tehuantepec, Oaxaca (Holthuis \& Villalobos 1962).

Las medidas de regulación actuales para este recurso incluyen una talla mínima de captura de 82,5 mm de longitud de cefalotórax (LC) para $P$. interruptus y $P$. inflatus en el Pacífico mexicano y el Golfo de California y $75 \mathrm{~mm}$ de LC para $P$. gracilis y $P$. inflatus, en los litorales del Pacífico mexicano correspondientes a Michoacán, Guerrero, Oaxaca y Chiapas. El periodo de veda comprende del 1 de julio al 30 de octubre a lo largo de la 
costa occidental del Golfo de California (D.O.F. 1993 ${ }^{1}$, $2005^{2}$ )

El estudio de la fecundidad como indicador del potencial reproductivo en $P$. inflatus es importante debido a que está relacionado con la capacidad de renovación de la población (Gracia 1985). Sin embargo, la fecundidad puede estar influenciada por factores externos tales como la cantidad y calidad del alimento, la temperatura y por características propias de la especie como las variaciones en las tallas, peso y edad (TapiaVázquez \& Castro-González 2000).

Entre los estudios que han sido realizados sobre la fecundidad en langostas espinosas en el Pacífico mexicano, destacan los de Pineda-Barrera et al. (1975), Ayala-Martínez \& Lucero-Telechea (1984) y Tapia-
Vázquez \& Castro-González (2000) para P. interruptus, así como Gracia (1985) para P. inflatus.

En este trabajo se estimaron la fecundidad, proporción de sexos y el índice de potencial reproductivo de $P$. inflatus en el Parque Nacional Bahía de Loreto con el fin de proporcionar información que ayude a comprender la capacidad de renovación de la población en el Golfo de California.

\section{Material y métodos}

De febrero de 2004 a enero de 2005, fue capturado un promedio de 35 ejemplares por mes de $P$. inflatus en el Parque Nacional Bahía de Loreto, Golfo de California, México, $\left(26^{\circ} 4^{\prime} 17^{\prime \prime} \mathrm{N}, 111^{\circ} 4^{\prime} 34^{\prime \prime W}\right.$ a $25^{\circ} 40^{\prime} 22^{\prime \prime} \mathrm{N}$, $111^{\circ} 40^{\prime} 22^{\prime \prime W}$ ) (Fig. 1). Las capturas se realizaron por

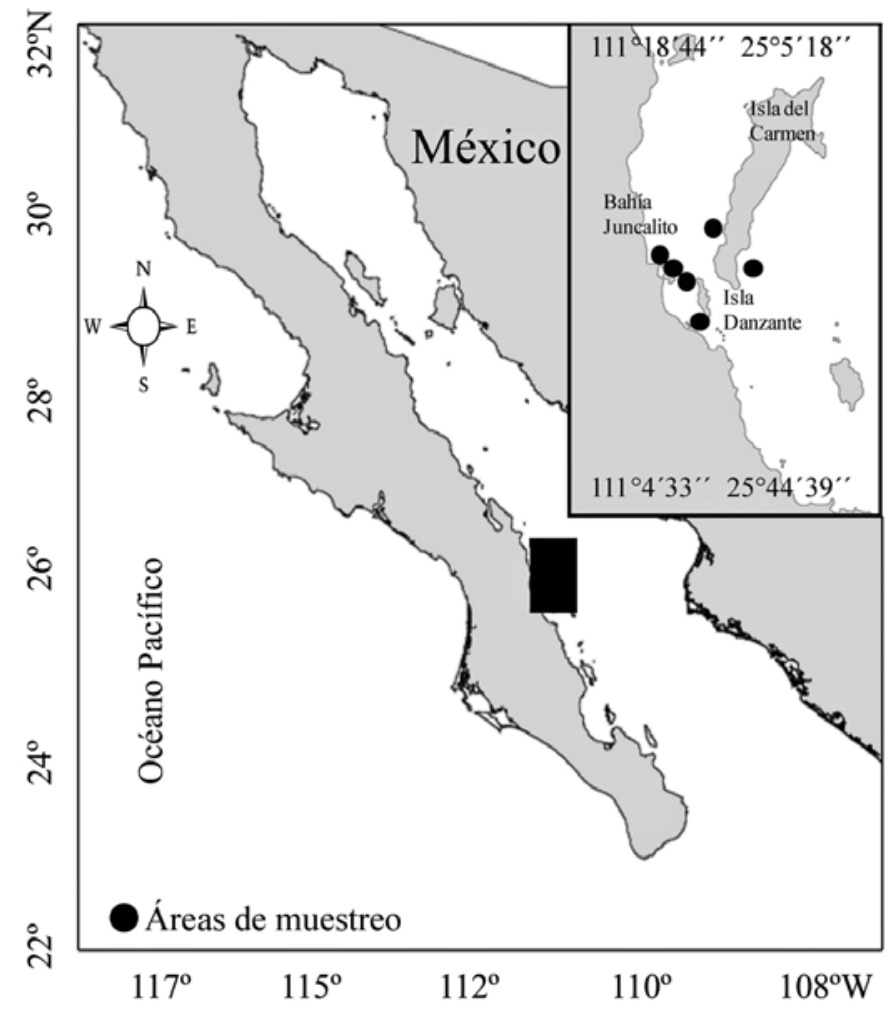

Figura 1

Área de estudio. El rectángulo negro indica la Bahía de Loreto, Golfo de California, México

Study area. The black rectangle indicates the Loreto Bay, Gulf of California, Mexico

${ }^{1}$ DOF. 1993. NOM-006-PESC-1993, acuerdo que regula el aprovechamiento de todas las especies de langosta de jurisdicción federal del Golfo de México y Mar Caribe, así como el Océano Pacífico mexicano, incluyendo el Golfo de California. Fecha de publicación 31-12-1993.

${ }^{2}$ DOF. 2005. Acuerdo por el que se modifican las épocas y zonas de veda de langosta azul (Panulirus inflatus), langosta verde ( $P$. gracilis) y langosta roja (P. interruptus) en aguas de jurisdicción federal del Océano Pacífico, incluyendo el Golfo de California. Fecha de publicación 31-12-1993. 
buceo libre durante la noche. Con el fin de que la captura fuera representativa de la abundancia relativa de la población, tres buzos recorrieron aproximadamente 1500 m paralelos a la costa entre 1 y 10 metros de profundidad, capturando todos los ejemplares de las langostas visibles utilizando arpones. De cada organismo se registró el sexo ( $\mathrm{H}=$ hembra, $\mathrm{M}=$ macho), presencia o ausencia de huevos en incubación, peso total (PT) y la longitud del cefalotórax (LC) medida desde la escotadura interorbital hasta el extremo posterior del cefalotórax.

Se determinó la proporción de sexos general, por mes y por intervalos de tallas. La significación estadística fue probada con un análisis de $\chi^{2}$ estableciendo como hipótesis nula una proporción de sexos $1 \mathrm{H}$ : $1 \mathrm{M}$, el valor observado fue comparado con el valor teórico de $\chi^{2}$ con un $95 \%$ de nivel de confianza (Sokal \& Rohlf 1979).

En el campo, las masas ovígeras fueron retiradas de las hembras e inmediatamente preservadas en formol neutro al 5\% preparado con agua de mar. En el laboratorio, los huevos fueron separados de los pleópodos utilizando cloro, deteniendo la reacción con tiosulfato de sodio (Choy et al. 1985, Villalejo-Fuerte et al. 2006). Posteriormente los huevos fueron colocados en un horno a $50^{\circ} \mathrm{C}$ y se removieron continuamente para que el secado fuera uniforme, hasta lograr peso constante.

Para estimar la fecundidad parcial, se pesaron tres submuestras de 0,01 g de cada masa ovígera; los huevos en cada submuestra fueron cuantificados manualmente con ayuda de un estereoscopio. La fecundidad parcial se determinó con la ecuación de Pineda-Barrera et al. (1975).

$$
F=\frac{G * N(X)}{g}
$$

donde: $\mathrm{F}$ = fecundidad parcial en número de huevos, $\mathrm{G}=$ peso total de la masa ovígera $(\mathrm{g}), \mathrm{N}(\mathrm{X})$ = número promedio de huevos de las tres submuestras, $\mathrm{g}=$ peso promedio de las tres submuestras (g).

Para analizar la relación entre la fecundidad parcial y la longitud del cefalotórax se ajustaron los datos a un modelo potencial.

La fecundidad relativa se obtuvo como el número de huevos por gramo de peso total de las hembras.

Para estimar el aporte de huevos a la población se calculó el índice de potencial reproductivo (IPR) propuesto por Kanciruk \& Herrnkind (1976) el cual determina el porcentaje de producción de huevos por grupos de talla y se considera el número de desoves durante la estación reproductiva y la frecuencia numérica de las hembras en los muestreos. IPR $=\left(\mathrm{A}_{\mathrm{i}} \times \mathrm{B}_{\mathrm{i}} \mathrm{C}_{\mathrm{i}}\right) / \mathrm{D}$, donde $\mathrm{A}_{\mathrm{i}}$ es la proporción del total de hembras en el $i$ ésimo intervalo de tallas, $\mathrm{B}_{\mathrm{i}}$ es la proporción de hembras ovígeras en el $i$-ésimo intervalo de tamaños corporales, $\mathrm{C}_{\mathrm{i}}$ es la fecundidad promedio en el i-ésimo intervalo de talla y D $(2180,69)$ es una constante estandarizada. Para esto, se dividieron las hembras en tres grupos de tallas de acuerdo a los criterios de Briones-Fourzán \& LozanoÁlvarez (1992) para P. inflatus: menores de $80 \mathrm{~mm}$ de LC con dos desoves al año, entre 80 y $110 \mathrm{~mm}$ de LC con cuatro desoves al año y mayores de $110 \mathrm{~mm}$ de LC con cuatro desoves al año.

\section{Resultados}

Se capturó un total de 567 organismos, 302 fueron machos y 265 hembras de las cuales 97 fueron ovígeras; de estas últimas 21,65\% fueron menores de $80 \mathrm{~mm}$ de LC, 72,16\% estuvieron entre 80 y 110 mm de LC, y 6,19\% fueron mayores de $110 \mathrm{~mm}$ de LC.

En general, la proporción de sexos fue de 1H:1,13M y no difirió significativamente de $1 \mathrm{H}: 1 \mathrm{M}\left(\chi^{2}, P>0,05\right)$. El análisis temporal mostró diferencias significativas en la proporción de sexos en los meses de marzo, mayo, junio y noviembre $\left(\chi^{2}, P<0,05\right)$ (Fig. 2).

Respecto a la proporción de sexos por tallas, se encontraron diferencias significativas en los intervalos 80-90 y $90-100 \mathrm{~mm} \operatorname{LC}\left(\chi^{2}, P<0,05\right)$, en los que predominaron las hembras (1,68H:1M y 2,03H:1M) (Fig. 3). También se encontró diferencia significativa en la proporción de sexos de los organismos de mayor talla, en los intervalos $110-120$ hasta $150-160$ y 170-180 mm de LC, en los que predominaron los machos $\left(\chi^{2}, P<0,05\right)$.

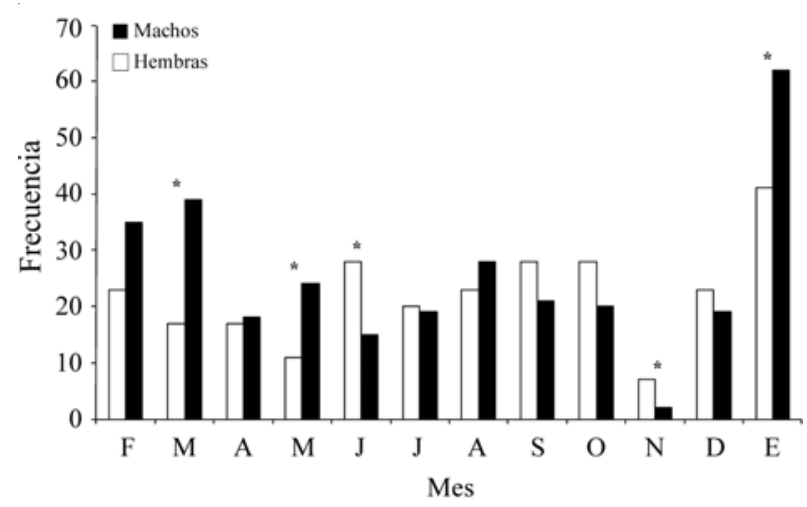

Figura 2

Proporción de sexos por mes en Panulirus inflatus. Los asteriscos indican diferencias estadísticamente significativas

Monthly sex ratio of Panulirus inflatus. The asterisks indicate differences statistically significant 


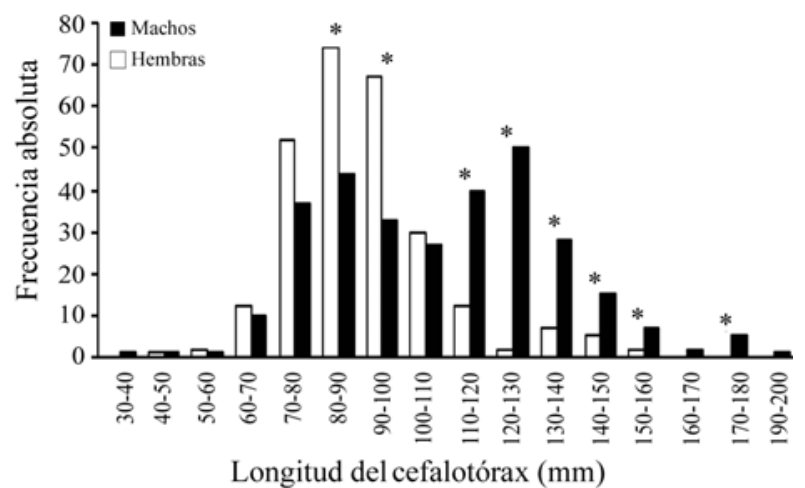

Figura 3

\section{Proporción de sexos por intervalos de talla de $10 \mathrm{~mm}$ en Panulirus inflatus. Los asteriscos indican diferencias estadísticamente significativas}

Sex ratio of Panulirus inflatus by $10-\mathrm{mm}$ size intervals. The asterisks indicate differences statistically significant

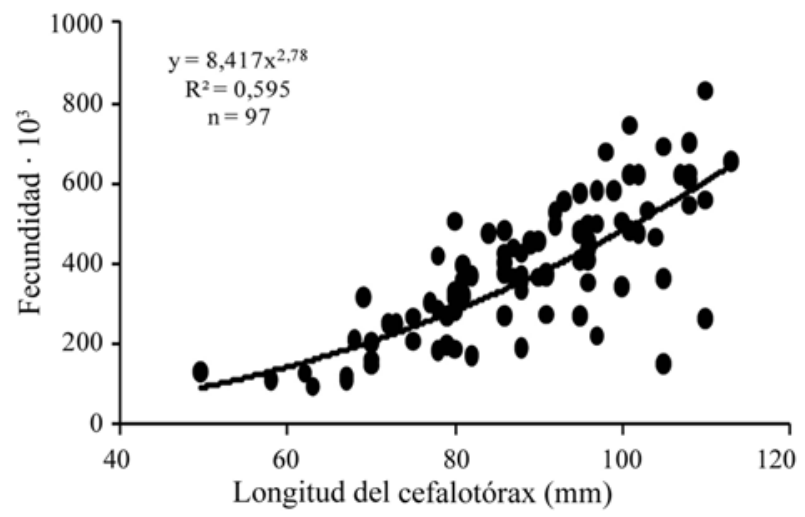

Figura 4

\section{Variación de la fecundidad con relación a la talla en Panulirus inflatus}

Fecundity variation in relation to size of Panulirus inflatus

Tabla 1

Variación estacional en la fecundidad de Panulirus inflatus y su relación con la longitud cefalotorácica (LC, mm)

Seasonal variations in fecundity of Panulirus inflatus and its relationship with carapace length (LC, mm)

\begin{tabular}{|c|c|c|c|c|c|c|}
\hline \multirow{2}{*}{ Estación } & \multirow{2}{*}{ LC media $(\mathrm{mm})$} & \multirow{2}{*}{$\begin{array}{c}\text { Fecundidad media } \\
\left(\mathrm{N}^{\circ} \text { huevos }\right)\end{array}$} & \multicolumn{2}{|c|}{ Relación Fecundidad-LC } & \multirow{2}{*}{$\mathrm{R}^{2}$} & \multirow{2}{*}{$\mathrm{n}$} \\
\hline & & & a & b & & \\
\hline Primavera & 87,02 & 383.533 & 92,600 & 1,849 & 0,556 & 24 \\
\hline Verano & 86,00 & 353.874 & 2,143 & 2,681 & 0,675 & 45 \\
\hline Otoño & 92,00 & 433.904 & 2,366 & 2,659 & 0,530 & 28 \\
\hline Invierno & \multicolumn{6}{|c|}{ No se capturaron hembras ovígeras } \\
\hline
\end{tabular}

La fecundidad varió entre 94.837 y 830.149 huevos, con una media ( \pm desviación estándar) de $384.314 \pm$ 162.147 huevos (Fig. 4). La fecundidad relativa promedio fue de 952 huevos $\mathrm{g}^{-1}$ de peso de las hembras y la desviación estándar de 222 huevos g-1

Las hembras de $90 \mathrm{~mm}$ de LC fueron capturadas durante primavera y verano con fecundidades promedios de 383.533 y 353.874 huevos respectivamente, mientras que las hembras mayores a $90 \mathrm{~mm}$ de LC se recolectaron principalmente en otoño y presentaron una fecundidad media de 433.904 huevos (Tabla 1).

El índice de potencial reproductivo indicó que las hembras menores a $80 \mathrm{~mm}$ de LC aportaron el 10,82\% de los huevos producidos durante la estación reproductiva, las hembras de 80 a $110 \mathrm{~mm}$ de LC aportaron el 80,23\% y las hembras con tallas mayores a $110 \mathrm{~mm}$ de LC aportaron solamente el 8,95\% de la producción de huevos en la zona de estudio (Tabla 2). La constante D $(2180,69)$ se tomó como el intervalo de tallas más productivo (en este caso 100-110 mm de LC) el cual se estandarizó como el 100\% (Kanciruk \& Herrnkind 1976).

\section{Discusión}

La proporción de sexos puede variar en las langostas espinosas. Briones-Fourzán \& Contreras-Ortiz (1999) señalan que las hembras de $P$. argus en Quintana Roo, México, pueden obtenerse en menor proporción debido posiblemente al método de muestreo utilizado. Aunque en general, para $P$. inflatus en el Parque Nacional Bahía de Loreto no se encontró diferencia significativa en la proporción de sexos, sí hubo una proporción mayor de machos a partir de los $110 \mathrm{~mm}$ de longitud cefalotorácica. La variación en la proporción sexual en $P$. guttatus ha sido atribuida a movimientos entre sexos durante el 
Tabla 2

Índice de potencial reproductivo para las hembras de Panulirus inflatus en el Parque Nacional Bahía de Loreto. $E$ es el porcentaje del total de huevos y $F_{i}=E_{i} / A_{i}$ es la productividad de huevos específica en el $i$-ésimo intervalo de tallas

Potential reproductive index to female of Panulirus inflatus at National Park Bahía de Loreto. E is the percentage of total eggs and $F_{i}=E_{i} / A_{i}$ is the eggs productivity specified in $i$ - th size range

\begin{tabular}{|c|c|c|c|c|c|c|c|c|c|c|c|}
\hline $\mathrm{LC}(\mathrm{mm})$ & $\begin{array}{l}\mathrm{N}^{\circ} \text { total } \\
\text { de } \\
\text { hembras }\end{array}$ & $\begin{array}{c}\mathrm{N}^{\circ} \\
\text { hembras } \\
\text { ovígeras }\end{array}$ & A & B & $\begin{array}{l}\text { Fecundidad } \\
\text { promedio } \\
(\mathrm{C} \times 1000)\end{array}$ & $\begin{array}{l}\mathrm{N}^{\circ} \text { de } \\
\text { desoves } \\
\text { al año }\end{array}$ & $\begin{array}{c}\text { Fecundidad } \\
\text { anual } \\
(\mathrm{C} \times 1000)\end{array}$ & IPR & Huevos año-1 & $\mathrm{E}$ & $\mathrm{F}$ \\
\hline $50-60$ & 3 & 2 & 0,75 & 66,67 & 120,70 & 2 & 241,41 & 2,79 & 482,82 & 0,32 & 0,43 \\
\hline $60-70$ & 12 & 6 & 2,26 & 50,00 & 163,42 & 3 & 490,28 & 8,49 & $2.941,71$ & 1,97 & 0,87 \\
\hline $70-80$ & 48 & 13 & 4,91 & 27,08 & 247,18 & 4 & 988,74 & 15,06 & $12.853,66$ & 8,64 & 1,76 \\
\hline $80-90$ & 75 & 28 & 10,57 & 37,33 & 351,53 & 4 & $1.406,12$ & 63,60 & $39.371,63$ & 26,46 & 2,51 \\
\hline $90-100$ & 67 & 24 & 9,06 & 35,82 & 445,87 & 4 & $1.783,04$ & 66,34 & $41.020,09$ & 28,45 & 3,14 \\
\hline $100-110$ & 30 & 18 & 6,79 & 60,00 & 535,01 & 4 & $2.140,04$ & 100,00 & $36.380,74$ & 24,45 & 3,82 \\
\hline$>110$ & 30 & 6 & 2,26 & 20,00 & 560,81 & 4 & $2.243,24$ & 11,65 & $15.702,74$ & 8,95 & 3,95 \\
\hline TOTAL & 265 & 97 & & & & & & & & & \\
\hline
\end{tabular}

periodo de actividad reproductiva (Losada et al. 2001), variaciones en abundancias debido a mortalidad diferencial por sexos (Evans et al. 1995) o bien que las hembras presentan una tasa de crecimiento menor que los machos (Briones-Fourzán \& Lozano-Álvarez 2003).

En este estudio, la fecundidad parcial osciló entre 94.837 y 830.149 huevos. Gracia (1985) reportó fecundidades de 69.100 a 570.786 huevos para $P$. inflatus en la costa centro-sur del Pacífico mexicano, muy por debajo de los resultados del presente trabajo. Lo anterior puede ser explicado por las tallas de las hembras, las cuales fueron mayores en el Parque Nacional Bahía de Loreto. También es posible que las condiciones ambientales en cantidad y calidad del alimento sean suficientes y favorables en la zona del Parque Nacional Bahía de Loreto como para modificar la fecundidad, tal como lo señalan Pineda-Barrera et al. (1975) y Gracia (1985) para $P$. interruptus y $P$. inflatus en otras regiones del Pacífico mexicano.

Las hembras menores de $90 \mathrm{~mm}$ LC se encontraron ovígeras durante la primavera y el verano y las hembras mayores de $90 \mathrm{~mm}$ LC durante el otoño. Lo anterior coincide con lo informado por Villalejo-Fuerte \& Velázquez-Abunader (2007) en Bahía de Loreto para $P$. inflatus, quienes encontraron la mayor frecuencia de hembras grandes en etapa reproductiva durante el otoño, cuando la temperatura del agua alcanzó el nivel máximo y hembras pequeñas en reproducción durante primavera, mientras que en invierno se registran otros procesos biológicos como el crecimiento apoyado por altas frecuencias de mudas. Por otra parte, estos resultados difieren de lo reportado por Gracia (1985) para P. inflatus en el centro-sur del Pacífico mexicano, quien observó el desove de organismos grandes durante la primavera (> $62,6 \mathrm{~mm}$ LC) mientras que en el otoño desovaron las hembras más pequeñas ( $<55,67 \mathrm{~mm} \mathrm{LC}$ ).

La fecundidad relativa (el número de huevos producido por gramo de peso de las hembras) es una medida indirecta del esfuerzo reproductivo. Gracia (1985) indicó que la fecundidad relativa de $P$. inflatus oscila entre 720 y 978 huevos por gramo de peso de la hembra, este intervalo es amplio y podría sugerir alguna diferencia en la fecundidad relativa respecto a la talla. En nuestro trabajo no encontramos correlación entre la fecundidad relativa y la talla; lo anterior demuestra que tanto hembras grandes como pequeñas producen en promedio el mismo número de huevos por unidad de peso corporal sugiriendo que en $P$. inflatus la producción de huevos conlleva una inversión energética equivalente en hembras grandes y pequeñas.

El índice de potencial reproductivo muestra que el aporte de huevos de las hembras menores de $80 \mathrm{~mm}$ de LC es bajo (10,93\%); lo anterior se explica por una parte, por su baja fecundidad y por otra, por el menor número de desoves durante la temporada de reproducción (dos al año); este porcentaje coincide con lo reportado por Briones-Fourzán \& Contreras-Ortiz (1999). Las hembras entre 80 y $110 \mathrm{~mm}$ de LC desovan hasta cuatro veces al 
año (Briones-Fourzán \& Lozano-Álvarez 1992) y son la fracción más abundante en la población de Bahía de Loreto, por lo tanto aportan el mayor porcentaje de huevos (mas del 80\%). Por su parte, las hembras mayores de $110 \mathrm{~mm}$ de LC tienen fecundidad alta, alrededor de 800 mil huevos por hembra, y desovan hasta cuatro veces durante la temporada reproductiva, su aporte en número de huevos es bajo debido a que son poco abundantes en la población, probablemente la supervivencia de langostas grandes es afectada por factores ecológicos como la disponibilidad de alimento, depredación y enfermedades (Losada et al. 2001).

De acuerdo con estos resultados se concluye que, en el Parque Nacional Bahía de Loreto, las hembras de $P$. inflatus de 80 a $100 \mathrm{~mm}$ de LC aportan la mayor cantidad de huevos y por lo tanto de larvas en la región, este segmento de la población está considerado en las medidas de regulación por parte del sector oficial.

\section{Agradecimientos}

Al Instituto Politécnico Nacional (IPN) por proporcionar los fondos para el Proyecto SAPI 2009753. COFAA y EDI-IPN por las becas a Marcial Villalejo-Fuerte y Arturo Tripp-Quezada. Al Programa PIFI-IPN y CONACYT por las becas otorgadas a José Iván Velázquez-Abunader. A David Villalejo Fuerte y Manuel Zamarrón Núñez por su ayuda durante los muestreos. A Nilia Cuellar Araujo por su ayuda en la edición y traducción del resumen al idioma inglés. Los autores extienden sus agradecimientos a dos revisores anónimos por sus valiosos comentarios y sugerencias que ayudaron a mejorar el presente trabajo.

\section{Literatura citada}

Ayala-Martínez Y \& F Lucero-Telechea. 1984. Fecundidad de la langosta roja (Panulirus interruptus Randall) en la región de Bahía Magdalena y San Juanico, B.C.S., México. Ciencia Pesquera 4: 33-48.

Briones-Fourzán P \& G Contreras-Ortiz. 1999. Reproduction of the spiny lobster Panulirus guttatus (Decapoda: Palinuridae) on the Caribbean coast of México. Journal of Crustacean Biology 19: 171-179.

Briones-Fourzán P \& E Lozano-Álvarez. 1992. Aspects of the reproduction of Panulirus inflatus (Bouvier) and $P$. gracilis Streets (Decapoda: Palinuridae) from the Pacific coast of México. Journal of Crustacean Biology 12: 41-50.

Briones-Fourzán P \& E Lozano-Álvarez. 2003. Factors affecting growth of the spiny lobsters Panulirus gracilis and Panulirus inflatus (Decapoda: Palinuridae) in Guerrero, Mexico. Revista de Biología Tropical 51: 165-174.
Choy SC. 1985. A rapid method for removing and counting eggs from fresh and preserved decapod crustaceans. Aquaculture 48: 369-372.

Evans CR, APM Lockwood, AJ Evans \& E Free. 1995. Field studies of the reproductive biology of the spiny lobsters Panulirus argus (Latreille) and P. guttatus (Latreille) at Bermuda. Journal of Shellfish Research 14: 371-381.

Gracia AG. 1985. Variación estacional en la fecundidad de la langosta Panulirus inflatus (Bouvier, 1895) (Crustacea; Decapoda: Palinuridae). Ciencias Marinas 11: 7-27.

Holthuis LB \& A Villalobos. 1962. Panulirus gracilis Streets y Panulirus inflatus (Bouvier), dos especies de langosta (Crustacea, Decapoda) de la costa del Pacífico de América. Anales del Instituto de Biología, Universidad Nacional Autónoma de México 32: 251-275.

Kanciruk P \& F Herrnkind. 1976. Autumnal reproduction in Panulirus argus at Bimini, Bahamas. Bulletin of Marine Science 26: 417-432.

Losada TV, JM Posada \& F Losada. 2001. Size and reproductive status of fished spotted spiny lobster, Panulirus guttatus, in Morrocoy National Park, Venezuela: a preliminary report. Marine and Freshwater Research 52: 1599-1603.

Pineda-Barrera J, CAJ Díaz de León \& F Uribe-Osorio. 1975. Fecundidad de la langosta roja Panulirus interruptus (Randall, 1842) en Baja California. Ciencia Pesquera 1: 99-118.

SAGARPA. 2003. Anuario estadístico de pesca, 266 pp. Comisión Nacional de Acuacultura y Pesca, Secretaría de Agricultura, Ganadería, Desarrollo Rural, Pesca y Alimentación, México.

Sokal RR \& FJ Rohlf. 1979. Biometría, principios y métodos estadísticos en la investigación biológica, 887 pp. H. Blume, Nueva York.

Tapia-Vázquez OL \& JJ Castro-González. 2000. Fecundidad y anatomía microscópica del ovario de la langosta roja Panulirus interruptus de Punta Eugenia, BCS., México. Ciencia Pesquera 14: 63-66.

Villalejo-Fuerte M \& JI Velázquez-Abunader. 2007. Reproduction of the blue lobster Panulirus inflatus (Bouvier, 1895) at "Bahia de Loreto", National Park, Gulf of California. Journal of Shellfish Research 26: 1165-1168.

Villalejo-Fuerte M, M Ramírez-Rodríguez, EF Balart \& C Rodríguez-Jaramillo. 2006. Fecundity of Cancer johngarthi Carbacho, 1989 (Decapoda: Brachyura: Cancridae) from Southern Baja California's Western Coast, Mexico. Journal of Shellfish Research 25: 995-997. 